\title{
TU/e EmonOWEN

\section{On the set of obtainable reference trajectories using minimum variance control}

Citation for published version (APA):

Engwerda, J. C. (1985). On the set of obtainable reference trajectories using minimum variance control. (Memorandum COSOR; Vol. 8523). Technische Hogeschool Eindhoven.

Document status and date:

Published: 01/01/1985

\section{Document Version:}

Publisher's PDF, also known as Version of Record (includes final page, issue and volume numbers)

\section{Please check the document version of this publication:}

- A submitted manuscript is the version of the article upon submission and before peer-review. There can be important differences between the submitted version and the official published version of record. People interested in the research are advised to contact the author for the final version of the publication, or visit the $\mathrm{DOI}$ to the publisher's website.

- The final author version and the galley proof are versions of the publication after peer review.

- The final published version features the final layout of the paper including the volume, issue and page numbers.

Link to publication

\section{General rights}

Copyright and moral rights for the publications made accessible in the public portal are retained by the authors and/or other copyright owners and it is a condition of accessing publications that users recognise and abide by the legal requirements associated with these rights.

- Users may download and print one copy of any publication from the public portal for the purpose of private study or research.

- You may not further distribute the material or use it for any profit-making activity or commercial gain

- You may freely distribute the URL identifying the publication in the public portal.

If the publication is distributed under the terms of Article 25fa of the Dutch Copyright Act, indicated by the "Taverne" license above, please follow below link for the End User Agreement:

www.tue.nl/taverne

Take down policy

If you believe that this document breaches copyright please contact us at:

openaccess@tue.nl

providing details and we will investigate your claim. 
Memorandum COSOR $85-23$

On the set of obtainable reference trajectories using minimum variance contro1

by

J. Engwerda

Eindhoven, Netherlands 


\title{
On the set of obtainable reference trajectories using minimum variance control
}

\author{
J. Engwerda
}

\begin{abstract}
:
In this paper it is investigated what will be the set of reference trajectories that can be tracked when Minimum Variance control is used.

A necessary condition on the dynamic evaluation of a reference trajectory is deduced. For the case of a time-invariant system all the trackable reference trajectories are characterized exactly. The effect of limiting the control possibilities on the ability to track a certain reference trajectory is also discussed. The analyzed system is assumed to be linear, finite dimensional and discrete-time. The derived results are interpreted for economic systems.
\end{abstract}

\section{Introduction}

In the case that reference trajectories are used in the analysis of linear systems it is usually either assumed that these reference points are generated themselves by a linear system, or that they may vary randomly in time. Hardly any attention is paid to the question to what extent these assumptions can be justified.

In economics, for example, the introduction of a reference path is often motivated by saying that the government sets the goals. This is of course a trivial statement of fact.

It is however a rather naive strategy to take these goals for granted and then calculate an optimal control law, given some cost criterium for it. Telling the government that some control scheme is the optimal way to minimize the gap between target variables and its reference values makes no sense when this gap widens every time step. The government is then aiming at conflicting objectives.

The best a control engineer can do in such a case is to mention to the government all those reference paths that can be tracked ultimately together with the corresponding control efforts at which they can be obtained. Doing so the control engineer gives the government the relevant information it needs to come to a well-considered decision about its goal setting.

Wohltmann has shown in'a recent paper for continuous time-varying systems [6], that a necessary condition for linear systems to be able to track a random reference trajectory is that the number of inputs is equal to or greater than the 
number of variables to be tracked. This is a generalization of the Tinbergen condition. As Wohltmann points out himself, this is a rather stringent condition, and will not always be fulfilled in practice.

The result that Wohltmann derived, depends strongly on the definition of targetpath controllability. A system is called target-path controllable if for every reference path there exists a control function such that the system output will exactly be equal to the reference output at any time during a time interval $\left[t_{0}, t\right]$. For time independent, discrete time systems this subject has been investigated by Preston and Pagan in [5]. In this book they derive necessary and sufficient conditions for target-path controllability, using Silverman's structure algorithm.

In the present paper an attempt is made to link preference specification, on the one hand, and policy existence on the other. To this end, a much weaker definition of target-path controllability will be given, from which still very appealing conditions can be deduced which have to be satisfled for the reference trajectory in order to be target-path controllable. An additional advantage of this approach is that time varying systems can be analyzed in the same way as time invariant systems.

The rest of the paper is organized as follows. In section II the definition of weak target-path controllability and admissibility of a reference path will be given. After that a necessary condition for a reference trajectory to be admissible will be derived.

In section III the special case of time invariant systems will be considered. This section is divided into two subsections, namely section III-A in which the set of all admissible reference trajectories will be characterized exactly, and section III- $B$ where the effects of limiting the control possibilities on the admissibility property of a reference trajectory are discussed.

Section III is followed by a description of an alternative approach to model limited control possibilities. The advantage of such an alternative approach is that it can be treated in a much easier way with standard mathematical tools.

Before conclusions are drawn in the final section VI, in section $\mathrm{V}$ an economic interpretation is given of the results in the case that the base system represents an economic system.

\section{Definition and sufficient conditions for reference path admissibility}

The base system analyzed in this paper is described by the following linear, finite dimensional, time varying, difference equation.

$$
y_{k+1}=A_{k} y_{k}+B_{k} u_{k}+C_{k} x_{k}, \quad k=1,2, \ldots
$$

where $y_{k}$ is an n-dimensional output vector to be controlled and is observed in period $k ; u_{k}$ is an m-dimensional input/control vector with $m \leq n ; x_{k}$ is an $p$ dimensional uncontrollable deterministic input vector, called exogeneous noise 
and is assumed to be known at period $k$. The initial values of the system are $y_{0}=\bar{y}_{0}$ and $u_{0}=\bar{u}_{0}$. It is assumed that all matrices are bounded in time, and that the matrices $B_{k}$ are all full rank.

Without changing the essential results of this paper, the system may be extended by an additive white-noise vector $w_{k}$, with $\operatorname{cov}\left\{w_{k} w_{l}{ }^{7}\right\}=\sum_{w} \delta_{k l}$, where $\delta_{k l}$ is the Kronecker delta, and $T$ denotes the transpose of a matrix. Introduction of such a random vector will only give rise to notational complications, and for this reason it is abandoned here.

As pointed out in the introduction, the problem considered in this paper consists of determining the set of reference trajectories that can be tracked by a good choice of the control function. To cope with this problem we first have to define what we mean by tracking. This is formalized in the definitions of admissible reference trajectories and weak target-path controllability given below.

Assume that the base system is described by Equation (1), that the initial state of the system is $\bar{y}_{0}$, and that a cost criterium $J$ is given, which has to be minimized. Now, define the closed-loop error $e_{k}:=y_{k}-y_{k}^{*}$ as the difference between output and reference vector, when a control function minimizing the cost criterium $J$ is applied to the system.

\section{Definition:}

A reference trajectory is called admissible with respect to $J$ and $\bar{y}_{0}$, if there exists a control function, minimizing $J$, such that the corresponding error function $e(\cdot)$ converges to zero when $k$ tends to infinity.

Remark: From now on we shall omit $J$ and $\bar{y}_{0}$ when we talk about the admissibility of a reference trajectory if it is clear which cost criterium and initial state are meant.

\section{Definition:}

System (1) is called Weak-Target Path Controllable (WTPC) if there exists an admissible reference trajectory.

The definition of weak target-path controllability is in two ways a generalization of Wohltmann's definition.

First, for WTPC, not all reference trajectories need to be admissible. Second, in Wohltmann's context a reference trajectory is called admissible only if it can be tracked exactly during the considered time interval.

We shall now derive a condition that must be satisfied by a reference trajectory in order to be admissible. But before that first a lemma is needed, the cost criterium $\mathrm{J}$ has to be defined, and a definition of the orthogonal projection-error matrix has to be given.

The lemrna gives us a necessary condition on the additive noise component in a recursive linear error equation, when boundedness of error is desired. Since the proof is straightforward, it is omitted. 


\section{Lernma 1:}

A necessary condition for boundedness (respectively convergence to zero) of $\boldsymbol{e}_{\boldsymbol{k}}$ in the following system

$$
e_{k+1}=A_{k} e_{k}+u_{k} \text {, where } A_{k} \text { is bounded, }
$$

is that $u_{k}$ is bounded (respectively converges to zero).

As a government's aim will usually be to track its reference values as quick as possible, the following cost criterium will be considered:

$$
J_{k+1}=\min _{u_{k}} e_{k+1}^{T} e_{k+1}, \quad k=1,2, \ldots
$$

The pursued analysis can however be applied to other cost criteria in the same way.

In the next theorem use is made of the orthogonal projection-error matrix. Since the reader may not be familiar with this concept, we give the following definition, which can for instance be deduced from [2].

\section{Definition:}

Let $B$ be an $n \times m$-matrix, with $m \leq n$.

Then the orthogonal projection-error matrix $M:=M(B)$ of $B$ is defined as: $M=I-B\left(B^{T} B\right)^{-1} B^{T}$.

Theorem 1: If a reference trajectory $y_{k}^{*}$ is admissible, then there exist vector sequences $u(\cdot)$ and $v(\cdot)$, where $v_{k}$ converges to zero when $k$ tends to infinity, such that:

$$
y_{k+1}^{*}=A_{k} y_{k}^{*}+C_{k} x_{k}+B_{k} u_{k}+M_{k} v_{k}, \quad \text { where } M_{k}=M\left(B_{k}\right)
$$

Proof: By definition, we have the admissibility of a reference trajectory only if there exists a control minimizing the error equation $J_{k+1}$.

The optimal control for system (1) minimizing $J_{k+1}$ is:

$$
u_{k}^{\text {opt }}=-\left(B_{k}^{T} B_{k}\right)^{-1} B_{k}^{T}\left[A_{k} y_{k}+C_{k} x_{k}-y_{k}^{*}+1\right], \quad k=1,2, \ldots
$$

Substitution of this optimal control into the process Equation (1) gives us a closed loop expression for the output vector $y_{k+1}$. The closed loop error $e_{k+1}$ is then given by:

$$
e_{k+1}=y_{k+1}-y_{k+1}^{*}=M_{k}\left(A_{k} y_{k}+C_{k} x_{k}-y_{k+1}^{*}\right) \text {. }
$$

This control-error equation can be rewritten as follows:

$$
e_{k+1}=M_{k} A_{k} e_{k}+M_{k}\left(A_{k} y_{k}^{*}+C_{k} x_{k}-y_{k+1}^{*}\right)
$$


From Lemma 1 it follows that a necessary condition for $e_{k}$ to converge to zero is the convergence of $M_{k}\left(A_{k} y_{k}^{*}+C_{k} x-y_{k+1}^{*}\right)$ to zero.

Since $M_{k} x$ is the projection error of vector $x$ on the image of matrix $B_{k}$, this condition can be rewritten as:

$$
y_{k+1}^{*}=A_{k} y_{k}^{*}+C_{k} x_{k}+B_{k} u_{k}+M_{k} v_{k} \quad \text { for some } u_{k} \text { and } v_{k}
$$

where $v_{k}$ converges to zero when $k$ tends to infinity.

\section{Corollary 1:}

From the proof of theorem 1 it follows that the control error equation can be written as:

$$
e_{k+1}=M_{k} A_{k} e_{k}+M_{k}\left(A_{k} y_{k}^{*}+C_{k} x_{k}-y_{k+1}^{*}\right)
$$

Theorem 1 leads to the important conclusion that all admissible reference trajectories must satisfy the property that they are generated in correspondence with the base system (1).

This is however generally not a sufficient condition. An additional assumption about matrix $M_{k} A_{k}$ that guarantees admissibility would be that at any time $k$, its singular values lie within the unit circle.

At the end of this section we want to link the result obtained here with the result obtained by Wohltmann.

\section{Corollary 2:}

Every reference path is admissible if and only if the number of control variables is equal to or greater than the number of target variables.

Proof:

From the theorem it is clear that in case the number of inputs is equal to the number of outputs, every reference path may be admissible. Since then matrix $M_{k}$ will be zero due to this assumption, it is seen from Corollary 1 that the control error equals zero for all. $k$.

So it can be concluded that every reference path is admissible in this case.

On the other hand, when the number of inputs is smaller than the number of outputs, it is clear from the theorem that not every reference trajectory will be admissible. Which completes the proof.

This result is an extension of the result derived by Preston and Pagan in [5] for time invariant systems, and fully agrees with Wohltmann's derived result for continues linear systems in [6]. 


\section{Time invariant systems}

III-A Characterization of the set of admissible reference trajectories

In this subsection we shall give a precise characterization of all admissible reference paths in case the system matrices $A_{k}, B_{k}$ and $C_{k}$ are all constant in time. For this purpose it is necessary to remember some well-known concepts and notation in systems theory, of which a summary is given below. For formal definitions and proofs the reader is referred to standard textbooks like [3]. [4] and [7].

Consider the system $x_{k+1}=A x_{k}+B u_{k}, x_{0}=\bar{x}_{0}$. Let $R_{0}$ be the set of states reachable from $x_{0}=0$. Then $R_{0}$ equals the linear subspace $\operatorname{Im} B+A \operatorname{Im} B+\cdots+A^{n-1} \operatorname{Im} B$. This subspace is usually abbreviated by $\langle A \mid B\rangle$.

It can then be proved that the set of all zero controllable initial states consists of $\left\{x_{0} \mid A^{n} x_{0} \in\langle A \mid B\rangle\right\}$. We will abbreviate this set by $\langle A \mid B\rangle_{0}$.

Furthermore, define the set of initial states $x_{0}$ such that the solution of $x_{k+1}=A x_{k}$ converges to zero when $k$ tends to infinity. We shall call this subspace the stable subspace and denote it by $x^{-}(A)$.

The following important property for the first mentioned system is well-known: $x_{0}$ is zero stabilizable (i.e. there exists a control function $u(\cdot)$ such that $x_{k} \rightarrow 0$, when $k \rightarrow \infty$ ) if and only if $x_{0}$ belongs to the subspace $x^{-}(A)^{k}+\langle A \mid B\rangle_{0}$.

Now let matrix $M$ be the orthogonal projection-error of matrix $B$, as defined in section III. Then with the help of the above mentioned results Theorem 2 can be proved.

\section{Theorem 2:}

A reference trajectory is admissible if and only if there exist $u(\cdot)$ and $\nu(\cdot)$ such that the following two conditions are met:

i) $\varepsilon_{0}:=y_{0}-y_{0}^{*}$, is zero stabilizable by means of $v(\cdot)$ in the following linear system:

$$
e_{k+1}=M A e_{k}-M v_{k}
$$

ii) $y_{k+1}^{*}=A y_{k}^{*}+B u_{k}+C x_{k}+M v_{k}$.

\section{Proof:}

$" \Rightarrow "$ From the definition of admissibility it follows that the control error

$$
e_{k+1}=M A e_{k}+M\left(A y_{k}^{*}+C x_{k}-y_{k+1}^{*}\right) \quad \text { (see Corollary 1) }
$$

converges to zero when $k$ tends to infinity.

This happens only if $M\left(A y_{k}^{*}+C x_{k}-y_{k+1}^{*}\right)$ converges to zero (Lemma 1).

But then $y_{k+1}^{*}$ satisfies the equation

$$
y_{k+1}^{*}=A y_{k}^{*}+B u_{k}+C x_{k}+M v_{k} \quad \text { for some vector } u_{k} \text { and } v_{k} \text {, }
$$


where $v$ converges to zero when $k$ tends to infinity.

Substitution of this expression into the control error equation yields:

$$
\begin{aligned}
e_{k+1} & =M A e_{k}-M v_{k}, \quad \text { where } v_{k} \rightarrow 0 \text { when } k \rightarrow \infty \\
e_{0} & =\bar{e}_{0} .
\end{aligned}
$$

So we have that $\vec{e}_{0}$ is zero stabilized by $v_{k}$, which completes this part of the proof.

$" \leftarrow "$ Equation ii) implies that $M\left(A y_{k}^{*}+C x_{k}-y_{k^{*}+1}^{*}\right)$ equals $-M v_{k}$ at any time $k$.

So the error equation can be rewritten as:

$$
\begin{aligned}
e_{k+1} & =M A e_{k}-M v_{k} \\
e_{0} & =\bar{e}_{0} .
\end{aligned}
$$

Since by assumption i) this vector sequence $v(\cdot)$ also stabilizes $\bar{e}_{0}$, it follows that a reference trajectory satisfying assumption i) and ii) will be admissible.

Remark: From the concepts defined in this section it is clear that an $\bar{e}_{0}$ stabilizing vector sequence $v(\cdot)$ will exist if and only if $\bar{e}_{0}$ is an element of the subspace $x^{-}(M A)+\langle M A| M>_{0}$.

\section{III-B Bounded control}

In the previous subsection, we characterized the set of admissible reference trajectories. However, no attention had been paid to the admissibility of the applied control. Since in economic reality it is often desired that the extent of control does not fluctuate too much, or even that the extent of control is bounded, we shall now model these restrictions and investigate what the effect will be on the admissibility property of a reference path.

Two ways to model these restrictions will be analyzed. One way to do this is to alter the cost criterium. This has the advantage that the newly created problem can easily be treated mathematically. This will be the subject of section IV.

The restrictions are modelled in this subsection as follows:

$$
\begin{aligned}
& \text { minimize } J_{k+1}:=\min _{u_{k}}\left\{e_{k}^{T} e_{k}\right\}, \text { given } d_{k m i n, i} \leq u_{k, i} \leq d_{k \max , i} \\
& \text { where } u_{k}=\left(u_{k, 1}, \ldots, u_{k, m}\right) \text { and } \\
& d_{k \min , i} \text { and } d_{k \max , i} \text { are given constants } i=1, \ldots, m .
\end{aligned}
$$


If the control possibilities are restricted for 1 consecutive time steps, the effect on the admissibility of a reference trajectory can be characterized exactly. Before properties concerning this subject are discussed, first some notation is introduced.

From now on $u_{k}^{a}$ will denote the applied control at time step $k$ and $u_{k}^{\text {opt }}$ will denote the optimal control at time step $k$ if there exists no bounds on the permitted extent of control at this time step.

Furthermore the difference between $u_{k}^{\alpha}$ and $u_{k}^{\text {opt }}$ is denoted by $\Delta u_{k}$, and denotes $e_{k_{0}}^{i q} t_{k}$ the control error at time $k_{0}+k$, when from time $k_{0}$ on for $q$ consecutive time steps application of optimal control was not possible.

The theorem now reads as follows:

\section{Theorem 3:}

If the control possibilities are restricted for 1 consecutive time steps, then a reference trajectory that is admissible in the sense of section III-A will remain admissible if and only if $B \Delta u_{k_{0}+l-1}+M A B \Delta u_{k_{0}+l-2}+\cdots+(M A)^{l-1} B \Delta u_{k_{0}}$ belongs to the stable space of matrix MA.

Here $k_{0}$ denotes the time at which the bounds first became effective.

Proof: It follows from the identity $y_{i+1}-y_{i+1}^{*}=A y_{i}+B u_{i}+C x_{i}-y_{i+1}^{*}$ that the equations

$$
e_{k_{0}^{+i}}^{(i)}=A y_{k_{0}+i-1}+B u_{k_{0}+i-1}^{a}+C x_{k_{0}+i-1}-y_{k_{0}+i}^{*}
$$

and

$$
e_{k_{0}+i}^{(i-1)}=A y_{k_{0}+i-1}+B u_{k_{0}+i-1}^{\mathrm{opt}}+C x_{k_{0}+i-1}-y_{k_{0}+i}^{*}
$$

hold.

Subtracting one equation from the other then yields:

(a) $e_{k_{0}+i}^{(i)}=B\left(u_{k_{0}+i-1}^{a}-u_{k_{0}+i-1}^{\text {opt }}\right)+e_{k_{0}+i}^{(i-1)}$.

Note that the admissible trajectories with respect to the cost criterium considered in this subsection are a subset of those considered in section III-A.

So, when after $i-1$ time steps the bounds on the extent of control disappear, we have from Theorem 2 that $e_{k_{0}+i}^{(i-1)}$ is equal to

(b) $M A e_{k_{0}+i-1}^{(i-1)}+M v_{k_{0}+i-1}$.

Substitution of (b) into (a) leads to the formula:

$e_{k_{0}+l}^{(l)}=B \Delta u_{k_{0}+l-1}+M A B \Delta u_{k_{0}+l-2}+\cdots+(M A)^{l-1} B \Delta u_{k_{0}}+e_{k_{0}+l}$,

where $e_{k_{0}+l}=e_{k_{0}+l}^{(0)}$. 
Since no restrictions exist anymore from time step 1 on, it is easily shown by induction that for $k$ greater than zero

$$
e_{k_{0}+l+k}^{(l)}-e_{k_{0}+l+k} \text { equals } M A\left(e_{k_{0}+l+k-1}^{(l)}-e_{k_{0}+l+k-1}\right)
$$

The last two equations imply the result as stated in the theorem.

The next recursive formula for the error $e_{k+n}^{(n)}$ can be derived from equation (a) and (b) in the proof of Theorem 3:

$$
e_{k+n}^{(n)}=M A e_{k+n-1}^{(n-1)}+B \Delta u_{k+n-1}+M v_{k+n-1 ;} ; \quad e_{k}^{(0)}=e_{k}
$$

This formula leads to the following results which hold if the bounds on the extent of control remain effective:

i) the system will not be weak target-path controllable

ii) if the eigenvalues of matrix $M A$ all lie within the unit circle and $\Delta u_{k}$ remains bounded, the error $e_{k}$ will remain bounded.

\section{Control cost}

As indicated in section III-B, instead of bounding the control here the effect of an alternative cost criterium on the reference path admissibility will be investigated. The criterium which is going to be used, is justified by the observation that a government often only has instruments at its disposal which are not allowed to deviate too much from their set points during a certain time period. Only when time passes these set points are allowed to change. These considerations give rise to the following $c$ ost criterium:

$$
\text { minimize } \tilde{J}_{k+1}:=\min _{u_{k}}\left\{e_{k}^{T} e_{k}+\left(u_{k}-u_{k}^{*}\right) R_{k}^{T} R_{k}\left(u_{k}-u_{k}^{*}\right)\right\}
$$

where $\left\{u_{k}^{*}, k=0,1,2, \ldots\right\}$ is the set point trajectory

of the control vector, and $R_{k}^{T} R_{k}$ is a positive

definite weighting matrix.

As in section II we shall also give a necessary condition here for the admissibility of a reference trajectory.

\section{Theorem 4:}

A reference path will be admissible for the base system (1) and cost criterium $J_{k+1}$ only if it is generated according to

$$
y_{k+1}^{*}=A_{k} y_{k}^{*}+C_{k} x_{k}+B_{k} u_{k}^{*}+v_{k}
$$

where $v_{k}$ converges to zero when $k$ tends to infinity. 
Proof: By straightforward differentiation of cost criterium $\tilde{J}_{k+1}$ it is seen that the optimal control for system (1) minimizing $\widetilde{J}_{k+1}$ is:

$$
u_{k}^{\text {opt }}=-\left(B_{k}^{T} B_{k}+R_{k}^{T} R_{k}\right)^{-1} B_{k}^{T}\left(A_{k} y_{k}+C_{k} x_{k}-y_{k}^{*}+1\right)+\left(B_{k}^{T} B_{k}+R_{k}^{T} R_{k}\right)^{-1} R_{k}^{T} R_{k} u_{k}^{*}
$$

Substitution of this optimal control into base system (1) leads to the following equation for the error $e_{x+1}$ :

$$
\mathbf{e}_{k+1}=\tilde{M}_{k} A_{k} y_{k}+\tilde{M}_{k} C_{k} x_{k}-\tilde{M}_{k} y_{k^{*}+1}^{*}+B_{k}\left(B_{k}^{T} B_{k}+R_{k}^{T} R_{k}\right)^{-1} R_{k}^{T} R_{k} u_{k}^{*}
$$

where $\tilde{M}_{k}$ equals $I-B_{k}\left(B_{k}^{T} B_{k}+R_{k}^{T} R_{k}\right)^{-1} B_{k}^{T}$.

After some matrix manipulation it can be shown that $B_{k}\left(B_{k}^{T} B_{k}+R_{k}^{T} R_{k}\right)^{-1} R_{k}^{T} R_{k} u_{k}^{*}$ is equal to $\tilde{M}_{k} B_{k} u_{k}^{*}$.

So the above error equation can be rewritten as:

$$
e_{k+1}=\tilde{M}_{k} A_{k} e_{k}+\tilde{M}_{k}\left(A_{k} y_{k}^{*}+C_{k} x_{k}+B_{k} u_{k}^{*}-y_{k+1}^{*}\right)
$$

From Lemma 1 we have as a consequence of the fact that $e_{k}$ must converge to zero, that $\tilde{M}_{k}\left(A_{k} y_{k}^{*}+C_{k} x_{k}+B_{k} u_{k}^{*}-y_{k+1}^{*}\right)$ must likewise converge to zero.

Since $R_{k}^{T} R_{k}$ is positive definite by assumption, we also have from Corollary 3 (below) that $\tilde{M}_{k}$ is positive definite.

In particular this means that $\tilde{M}_{k}$ is a symmetric matrix of full rank. This implies that $A_{k} y_{k}^{*}+C_{k} x_{k}+B_{k} u_{k}^{*}-y_{k}^{*}$ converges to zero, or to put it in another way:

$y_{k+1}^{*}$ equals $A_{k} y_{k}^{*}+C_{k} x_{k}+B^{k} u_{k}^{*}+v_{k}$.

where $v_{k}$ converges to zero.

In the proof of Theorem 4 use was made of the conjecture that if $R_{k}^{T} R_{k}$ is positive definite, then $\tilde{M}_{k}$ will also be positive definite. In the next lemma it is shown that all eigenvalues of matrix $\tilde{M}_{k}$ lie between zero and one. As a consequence, the correctness of the conjecture is immediate.

\section{Lemma 3:}

Let $R_{k}^{T} R_{k}$ be a positive definite matrix, and $\lambda$ an eigenvalue of $\tilde{M}_{k}$. Then the relation $0<\lambda \leq 1$ holds.

Proof: From the matrix inversion lemma (see e.g. [3]). it follows that $\tilde{M}_{k}$ is equal to $\left(I+B_{k}\left(R_{k}^{T} R_{k}\right)^{-1} B_{k}^{T}\right)^{-1}$.

Now $B_{k}\left(R_{k}^{T} R_{k}\right)^{-1} B_{k}^{T}$ is semi positive definite, so all eigenvalues of this matrix are equal to or greater than zero. But then all the eigenvalues of matrix $I+B_{k}\left(R_{k}^{T} R_{k}\right)^{-1} B_{k}^{T}$ are greater than or equal to one. So all the eigenvalues of matrix $\left(I+B_{k}\left(R_{k}^{T} R_{k}\right)^{-1} B_{k}\right)^{-1}$ lie between zero and one. This proves our lemma.] 


\section{Corollary 3:}

If $R_{k}^{T} R_{k}^{\prime}$ is positive definite, then $D_{k}$ will be positive definite.

Since the stability of the error equation is dominated by the behaviour of matrix $\tilde{M}_{k} A_{k}$, it would be nice if some general statements concerning the eigenvalues or singular values of this matrix could be made.

Due to the results of Lemma 3 this seems to be a manageable problem. One remark that has to be made in this context is that in general the eigenvalues and eigenvectors will not be situated within the unit circle. Take for example $R=I, B=\left(\begin{array}{l}1 \\ 0\end{array}\right)$ and $A=4 * I$. So the error equation will in general not be stable!

How this misbehaviour can be overcome is a question for future research.

\section{Economic Interpretation}

The aim of this section is to interpret some theorems, formulated and proved mathematically in the foregoing sections, in a setting of economic policy problems.

But first it should be pointed out that the conclusions drawn here depend on several assumptions about the model. Results obtained here therefore depend on the validity of these assumptions. The assumptions fall into a category of four types.

First we assumed that the economy can be described by a linear finite dimensional model, which possibly possesses an unmodelled part that is expressible as noise with zero expectation and finite variance.

Second, it was assumed that the structure of the economy is known to the government, and third that the exogeneous and reference values are also assumed to be known by the government. The fourth assumption concerns the fact that the government's aim is to track its target values as quick as possible.

Furthermore, it should be kept in mind that the most important results obtained in this paper are necessary conditions and not yet sufficient.

One of the results obtained in Section II was that if the number of control variables is equal to (or exceeds) the number of target variables, and these control variables can be applied independently, then every reference path can be tracked exactly. Consequently, if the goal of the government is to regulate the economy and some target variables seem to be uncontrollable, it may be possible for the government to realize its goal by introducing new independent control mechanisms.

So by introducing more buraucracy it might be possible for the government to regulate the economy as it wishes.

Introducing more buraucracy however brings with it more control cost for the government and has an impact on the welfare for the population. Taking this additional cost into account, it is seen from section IV that not all reference paths are obtainable anymore. So the effect of a good modelling of the 
government aims becomes clear.

We shall now give an interpretation of the sufficiency condition derived in Theorem 1 about the admissibility of a reference path. In this theorem it is observed that a reference path of a target variable must be generated in correspondence with the structural parameters prevailing in a country. It is seen that only the reference values of those target variables that can be directly influenced by the government can be updated intermediately. Moreover it is observed that for a small country - where the in fluence of the exogeneous variables on the national economy is large (like the Netherlands) - the government depends severely on what happens in the foreign countries in its choice of reference trajectories.

Note that all these conclusions only hold when no attention is paid to resulting additive control cost. Taking this cost into account. Theorem 4 can be used to derive results.

A sudden calamity in a foreign country, such as an oil crisis will effect the national economy of a small country, according to the foregoing developed theory, as follows.

At the start of such a calamity the economy will be disrupted. Due to the enormous influence of exogeneous variables on the admissible reference trajectories, several target variables will appear not to be obtainable anymore, unless the government takes radical measures. As time goes on, substitution effects will take place, which will lead to changes in the structural parameters. In course of time the government then has to choose new reference trajectories for its targets depending on the new structural .

parameters. These will probably differ from those before the calamity took place.

Note that in this theory substitution effects can only give rise to a change in structural parameters. Possible relations which might exist between parameters and target variables are not taken into account. By taking these relations into account, we have as a consequence that our assumption considering linearity of the model will no longer hold. In that case non-linear models have to be studied, which will be a subject for future research.

It is clear that the infuence of a calamity on the admissible reference paths of a large country - where the influence of the exogeneous variables on the economy is of minor importance (like the U.S.) - will be less severe.

The question now arises what a government can do if it rejects any admissible reference trajectory for a target variable. From Theorem 1 it is clear that the only possibility left is then to change the structural parameters (i.e, if the assumption made about the changes in structural parameters as stated above holds). This will be a question of long term policy. Two examples of the implications of this conclusion will now be given.

Suppose the economies of the Western European countries satisfy the model assumptions as stated above. Now it is generally agreed on that the high unemployment rates in these countries are due to a number of changes in the structural parameters in these countries in the last decade. So, if all involved 
countries abuse an increase of their government expenditures, according to this theory the only way left open to attack the problem is to pursue a policy that is fixed on changing other structural parameters

A policy that might have prevented these troubles is one in which the government acts as a structural buffer. This means that on the long run the government takes care that the desired goals are obtainable and that financial resources are stocked in order to overcome short term discrepancies.

Another interesting example of this theory is that developing countries benefit most from help that aimes at the improvement of structural parameters. The ultimate effect of such help will be that these countries can obtain a faster selfsustained growth of their economies. In general it can be said that all countries benefit from cooperation, since this will help to prevent each national economy in question for non-anticipated foreign shocks.

At the end of this section we recall the statement proved in Theorem 4 , that if a set point trajectory exists for the control to be applied, the admissible reference trajectories depend strongly on this set point sequence.

\section{Conclusions}

The objective of this paper was to give: a characterization of all reference paths that can be tracked by means of Minimum Variance control. A reference trajectory that could be tracked was called admissible.

It was proved that the admissible trajectories show a great correspondence with the base system. For time independer: systems all admissible reference trajectories were characterized exactly.

After that the effect of bounding the control on the admissibility property of a reference path for these systems was investigated. It appeared that when the bounds only existed for a finite time inierval, this effect could be traced exactly. When an infinite time horizon was considered, it was proved that under an additional assumption the admissibility property was almost preserved.

Since restrictions on the extent of control can be motivated by other reasons, the set of admissible trajectories was characterized for an alternative cost criterium. The necessary admissibility condition proved to be dependent on the control set point trajectory in this case.

As a special case the Tinbergen condition, "number of control variables $\geq$ number of target variables", proved to be extendable to discrete linear time dependent systems.

At the end of the paper some economic interpretations of the derived results were given. 


\section{REFERENCES}

[1] Aalders L., Engwerda J.C., and Otter P.W. (1983), Selftuning control of a macro economic system Proceedings of the 4th IFAC/IFORS/IIASA on Economic Dynamics and Control Washington D.C., U.S.A., 1984.

[2] Basilevsky A. (1983), Applied Matrix Algebra in the Statistical Sciences, pp.163 North Holland, New York.

[3] Kailath T. (1980), Linear Systems, pp.656 Prentice Hall.

[4] Kwakernaak H., and Sivan R. (1972), Linear Optimal Control Systems, chapter 6 Wiley, New York.

[5] Preston A.J., Pagon A.R. (1982), The Theory of Economic Policy, pp.237.262264,314,334 Cambridge University Press, New York.

[6] Wohltmann H.W. (1985), Target Path Controllability of Linear Time-Varying Dynamical Systems I.E.E.E. AC-30, pp.84-87.

[7] Wonham W.M. (1974), Linear Multivariable Control: a Geometric Approach, pp.36,37,54 Springer Verlag, Berlin. 